\title{
Parallel Coupled Microstrip Filters With Ground-Plane Aperture for Spurious Band Suppression and Enhanced Coupling
}

\author{
Maria del Castillo Velázquez-Ahumada, Jesús Martel, and Francisco Medina, Senior Member, IEEE
}

\begin{abstract}
Parallel coupled microstrip sections with a slotted ground plane are proposed as building blocks of coupled-line microstrip filters with enhanced performance. It is shown that, by proper adjustment of the ground-plane slot dimensions, the double frequency spurious band associated with unequal even/odd electrical lengths can be suppressed or meaningfully reduced. As an additional feature, this simple design relaxes tolerances of strip width and spacing in those cases where tightly coupled high-impedance sections are required. A rough preliminary design can be obtained within a few seconds using a fast optimization algorithm based on a quasi-TEM analysis of the coupled sections. Fine tuning is based on the use of a commercial electromagnetic simulator. Finally, experimental check of filter performance is provided.
\end{abstract}

Index Terms-Coupled-line microstrip filters, slotted ground plane, spurious response suppression.

\section{INTRODUCTION}

$\mathbf{P}$ ARALLEL coupled-line microstrip filters (PCMFs) are usual components in microwave integrated circuits owing to their simple design and versatility. However, when implemented in their traditional form on a single-layer substrate in microstrip technology, the filter developed by Cohn in 1958 [1] inherently presents two relatively serious drawbacks. The first one is the existence of a spurious passband at $2 f_{0}$ (where $f_{0}$ is the central frequency of the filter). This undesired passband is associated to the nonhomogeneous nature of the dielectric surrounding the conductors (this causes even and odd modes supported by the coupled line to have different phase velocities). The second problem comes from the difficulty of fabricating wide-band filters because of the weak coupling between the lines in the conventional structure [see Fig. 1(a)]. Strong coupling required by some filter specifications leads to very small values of strip width $w$ and strips spacing $s$, which cannot sometimes be accurately achieved in practice. Due to the practical importance of the aforementioned problems,

Manuscript received July 28, 2003; revised October 9, 2003. This work was supported by the Spanish Ministry of Science and Technology/European Union Fondo Europeo para el Desarrollo Regional funds under Project TIC2001-3163 and by the Spanish Junta the Andalucía.

M. del Castillo Velázquez-Ahumada and F. Medina are with the Grupo de Microondas, Departamento de Electrónica and Electromagnetismo, Facultad de Física. Universidad de Sevilla, 41012 Seville, Spain (e-mail: casvelahu@alum.us.es; medina@us.es).

J. Martel is with the Grupo de Microondas, Departamento de Física Aplicada 2, Escuela Tecnica Superior de Arquitectura, Universidad de Sevilla, 41012

Seville, Spain (e-mail: martel@us.es).

Digital Object Identifier 10.1109/TMTT.2004.823593

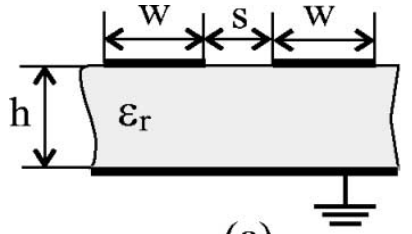

(a)

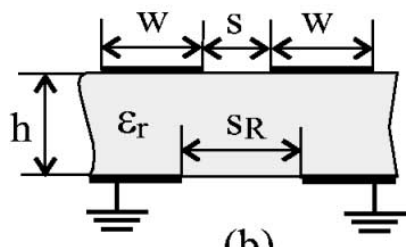

(b)

Fig. 1. Cross sections of basic building blocks of microstrip coupled lines for filter design. (a) Conventional. (b) Modified with ground-plane aperture.

a number of methods have been proposed in the literature to improve the out-of-band performance of PCMFs (apart from the obvious substitution of PCMFs by other types of microstrip filters not exhibiting those intrinsic drawbacks [2], although maybe showing other limitations). Roughly speaking, most of the methods available in the literature to suppress the undesired passband of PCMFs fall into one of the following two categories: those based on lumped load compensation [3], [4] and those based on modified structures with intrinsically equal modal phase velocities [5], [6]. Recent proposals, which can be considered sophisticated implementations of a mixing of those approaches, are the use of continuously varying (nonuniform) coupled transmission lines [7] or corrugated coupled microstrip lines [8]. Completely different approaches are also available. Thus, extra transmission zeros associated to extra coupling capacitances are used in [9] and [10], the utilization of a two-dimensional (2-D) photonic-bandgap ground plane is proposed in [11], and a periodic (sinusoidal) modulation of the coupled lines width is reported in [12].

Our proposal in this paper is to use the modified structure in Fig. 1(b), which simply incorporates in the well-known microstrip line a centered slot at the ground plane. The slot width $s_{R}$ can be adjusted so as to tune the even/odd phase velocities in order to suppress the spurious band. As an additional advantage, this structure provides tight coupling (in comparison with conventional microstrip lines), thus, relaxing the requirements on physical dimensions $w$ and $s$ in those cases where tight coupling is necessary [13]. This structure was analyzed by Masot $e t a l$. in [14] as a suitable configuration for high-directivity and/or 
high-coupling printed directional-coupler applications. In [14], a fast algorithm for perfect phase velocity matching (based on the judicious choice of $s_{R}$ ) was proposed in order to improve the directivity of the coupler. This algorithm is also used in our case as a first step in the design process. This step is carried out very quickly since the computer code providing the modal parameters of the basic coupled section was highly optimized [15] (the code is based on an accelerated quasi-static spectral-domain analysis). This is an additional advantage of our proposal when compared with other approaches requiring electromagnetic (EM) simulation since the beginning of the design process. Nevertheless, EM simulations are also necessary in our scheme for final fine tuning. The reason is that the end effects are important and quite different for even and odd modes when the coupled sections in Fig. 1(b) are used as building blocks of a PCMF. Note that overall electrical lengths, rather than modal phase velocities, must be matched for spurious band suppression and that end effects meaningfully contribute to this electrical length. Two different strategies have been employed in this paper to achieve electrical-length equalization. The first one (illustrated by cases A and B in Section II) consists of selecting $s_{R}$ so as to compensate differential end effects, i.e., $s_{R}$ is chosen not to achieve identical mode phase velocities, but to equalize overall modal electrical lengths (including end effects). The second strategy (case C) consists in retaining the value of $s_{R}$ that provides phase velocity matching along most of the extension of the coupled section, while local perturbations (widening of the slots) at the ends of the coupled sections are introduced to compensate for the difference between equivalent end effect modal electrical lengths. Experimental confirmation of the proposed methods is finally provided.

\section{FILTER DESIGN}

\section{A. One-Pole Butterworth Filter of $\Delta=20 \%$}

Let us consider the simple case of a Butterworth bandpass filter centered at $f_{0}=2 \mathrm{GHz}$ having a bandwidth of $\Delta=20 \%$ and order $N=1$ in order to illustrate the use of the block in Fig. 1(b). Following [1], we obtain for the two identical sections of the filter, $Z_{\text {even }}=77.67 \Omega$ and $Z_{\text {odd }}=38.04 \Omega$. The corresponding physical dimensions of the conventional and slotted ground filters are shown in Table I, as well as the even $(e)$ and odd $(o)$ modal effective permittivities $\epsilon_{e f}^{e}$ and $\epsilon_{e f}^{o}$. This design has been carried out in seconds on the basis of the quasi-static approach in [15] and the use of an ad hoc optimization scheme [14]. The optimization strategy takes into account that mode impedances are mainly controlled by strip width $w$ and strips separation $s$, while phase velocity mismatching is mainly affected by the ground slot width $s_{R}$. Thus, instead of using a general-purpose gradient optimization method, we only change one of the structural variables, each time attending only to the associated error function. If this scheme is repeated a few times, a very good solution is attained. Many more evaluations of the coupled-line parameters are required if the general-purpose optimization algorithm is used. From Table I, it can be observed that, as was predicted, both $w$ and $s$ are larger for the modified geometry than for the conventional one. This feature relax tolerances in the definition of linewidth and line separation when an accurate fabrication technique is not available.

TABLE I

Dimensions (In MiLlimeters) AND EFFeCtive PermitTivities of CONVENTIONAL AND MODIFIED COUPLED-Line BANDPASS FILTER A. SUBSTRATE THICKNESS: $0.635 \mathrm{~mm}$; PERMITTIVITY: $10 \epsilon_{0}$
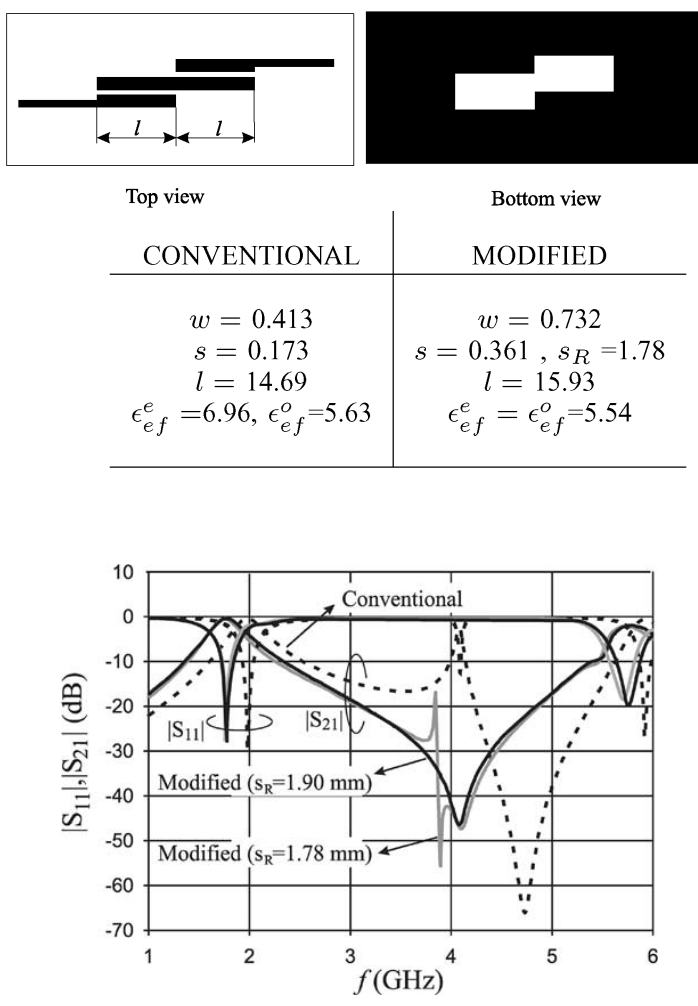

Fig. 2. Full-wave simulations obtained with Ensemble for the conventional filter of Table I (dashed line), the modified version with equal-mode phase velocities, i.e., with $s_{R}=1.78 \mathrm{~mm}$ (gray line), and the modified version with $s_{R}=1.90 \mathrm{~mm}$ for modal electrical lengths compensation (solid line).

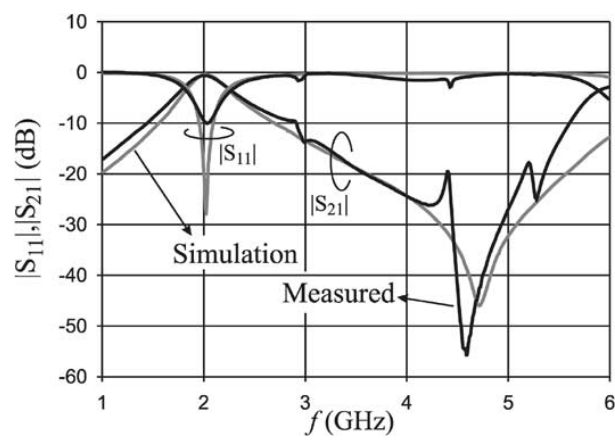

Fig. 3. Simulated and measured results for the final version of the filter design A.

Note that no edge effects were considered in this preliminary design. The physical lengths of the coupled sections were calculated following [16] (conventional filter) or using $l=\lambda_{e} / 4=\lambda_{o} / 4$ (modified filter), $\lambda_{e}$ and $\lambda_{o}$ being the even/odd wavelengths at $f_{0}$. These structures were simulated using the commercial package Ensemble and the obtained responses are shown in Fig. 2. As expected, the conventional filter presents spurious transmission at approximately $4 \mathrm{GHz}$. Since the central frequency matches pretty well the preliminary design prediction, we conclude that end effects are small in this case. However, owing to strong distortion of the surface current at the ground plane, these effects become important for the 
TABLE II

Dimensions (IN MiLlimeters) OF CONVENTIONAL AND MODIFIED BANDPASS PCMF B. The SAME SubStRate As IN A

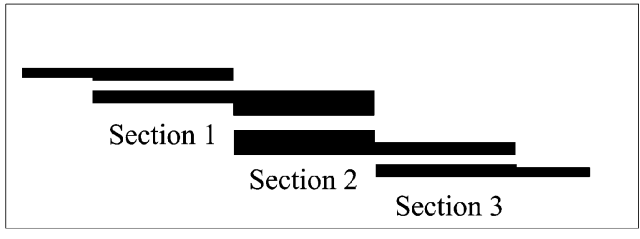

Top view

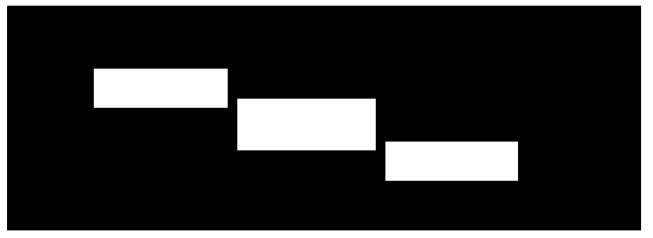

Bottom view

\begin{tabular}{l|c|c}
\hline Type of design & Sections 1,3 & Section 2 \\
\hline \hline Conventional & $w=0.184, s=0.0828, l=14.98$ & $w=0.238, s=0.0918, l=14.90$ \\
& $\epsilon_{e f}^{e}=6.52, \epsilon_{e f}^{o}=5.53$ & $\epsilon_{e f}^{e}=6.63, \epsilon_{e f}^{o}=5.54$ \\
\hline Modified with & $w=0.309, s=0.145, s_{R}=1.29$ & $w=0.404, s=0.165, s_{R}=1.65$, \\
$\epsilon_{e f}^{e}=\epsilon_{e f}^{o}$ & $l=15.96, \epsilon_{e f}^{e}=\epsilon_{e f}^{o}=5.52$ & $l=15.94, \epsilon_{e f}^{e}=\epsilon_{e f}^{o}=5.52$ \\
\hline Modified with & $w=0.412, s=0.194, s_{R}=1.80$, & $w=0.404, s=0.165, s_{R}=1.65$, \\
spurious band suppression & $l=15.85, \epsilon_{e f}^{e}=5.45, \epsilon_{e f}^{o}=5.00$ & $l=15.85, \epsilon_{e f}^{e}=\epsilon_{e f}^{o}=5.52$ \\
\hline
\end{tabular}

modified version of the filter, leading to a severe displacement of the central frequency. Moreover, this effect is different for each mode, in such a way that the equivalent excess lengths $\Delta l_{e}$ (even mode) and $\Delta l_{o}$ (odd mode) are not equal at all. As a consequence, the spurious band is not completely removed. This problem can be corrected by slightly increasing the slot width, as is also shown in Fig. 2 (solid line). The dimensions of $w$ and $s$ must be simultaneously adjusted so as to keep the same modal impedances, but this is not a problem thanks to the use of the fast quasi-static solver described in [15]. When $s_{R}=1.90 \mathrm{~mm}$, the small difference between $\epsilon_{e f}^{e}$ and $\epsilon_{e f}^{o}$ (note that the even mode is more affected than the odd mode when the slot width is augmented) compensates for the difference between $\Delta l_{e}$ and $\Delta l_{o}$. The spurious band then disappears.

As a final step, the length of the sections are slightly reduced so as to match the desired central frequency $(l=13.83 \mathrm{~mm}$ for this filter). The full-wave simulation of the final design is shown in Fig. 3. Measured results are also included in that figure. Discrepancies probably come from lack of accuracy in the fabrication process, but it is clear that the spurious band is meaningfully reduced with respect to the one expected from a conventional design. Interestingly, it should be mentioned that the final dimension of $s_{R}$ is not critical, which is in contrast with other approaches [6].

\section{B. Two-Pole Chebyshev Filter of $\Delta=30 \%$ and Ripple $0.1 \mathrm{~dB}$}

As a second example to illustrate additional details of the design procedure, we have designed a $\Delta=30 \%$ bandwidth Chebyshev bandpass filter with $f_{0}=2 \mathrm{GHz}$, order $N=2$, and ripple $0.1 \mathrm{~dB}$. Classical design requires $Z_{\text {even }}=115.3 \Omega$ and $Z_{\text {odd }}=40.57 \Omega($ Sections 1 and 3$)$, and $Z_{\text {even }}=103.7 \Omega$ and $Z_{\text {odd }}=38.64 \Omega$ (Section 2$)$. Dimensions of conventional, modified with identical modal velocities, and modified for end-effect compensation are shown in Table II. In this filter design, a detail deserves to be clarified. Note that, in this case, there are two different coupled sections. To obtain the final dimensions, we have used Ensemble to suppress the spurious band in the response of the one-pole filter that could be constructed based on each section separately. It can be

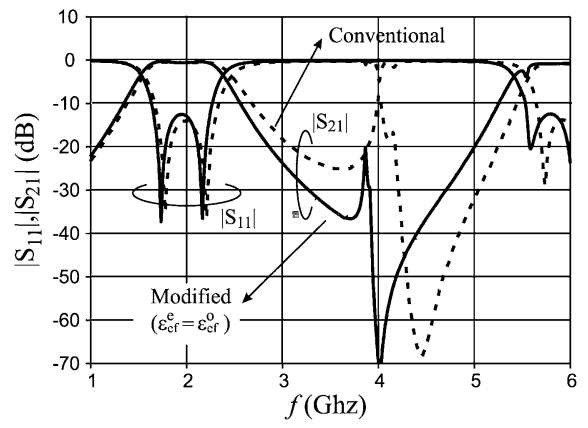

Fig. 4. Ensemble simulations for the conventional filter of Table II (dashed line) and the modified version (solid line) when $\epsilon_{e f}^{e}=\epsilon_{e f}^{o}$.

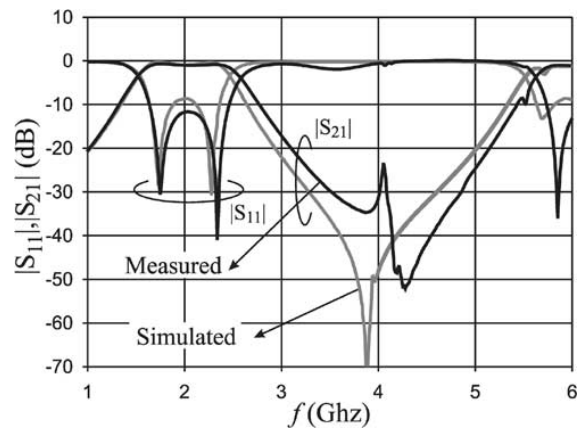

Fig. 5. Comparison between simulated and measured results for the final version of the designed filter $\mathrm{B}$.

observed that no relevant changes are required for the central section of the filter. We would like to emphasize again that the small values of $s$ required by the conventional design are not required by the filter based on the slotted structure. This permits to push further on the limitations on specifications associated with the line precision achievable with the available technology.

An important practical issue is that the slot under each section must be separated from the adjacent one (see the figure atop Table II) to avoid slot mode propagation. If the printed metal bridges separating the slots are eliminated, the filter response 
TABLE III

DIMENSIONS (IN MILLIMETERS) OF CONVENTIONAL AND MODIFIEd COUPLED-Line BANDPASS FILTER C

\begin{tabular}{l|c|c}
\hline Type of design & Sections 1, 3 & Section 2 \\
\hline \hline \multirow{3}{*}{ Conventional } & $w=0.357, s=0.134$, & $w=0.472, s=0.240$, \\
& $l=14.74$ & $l=14.63$ \\
& $\epsilon_{e f}^{e}=6.86, \epsilon_{e f}^{o}=5.60$ & $\epsilon_{e f}^{e}=7.07, \epsilon_{e f}^{o}=5.69$ \\
\hline \multirow{3}{*}{ Modified } & $w=0.620, s=0.263$, & $w=0.865, s=0.551$, \\
& $s_{R}=1.59, l=15.93$, & $s_{R}=2.09, l=15.96$, \\
& $\epsilon_{e f}^{e}=\epsilon_{e f}^{o}=5.54$ & $\epsilon_{e f}^{e}=\epsilon_{e f}^{o}=5.52$ \\
\hline
\end{tabular}

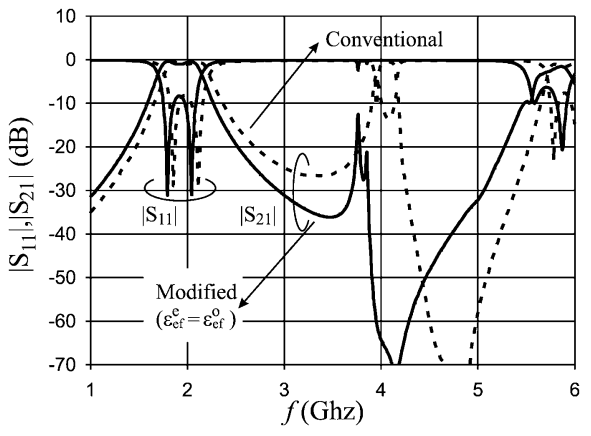

Fig. 6. Full-wave simulations obtained from Ensemble for the conventional filter of Table III (dashed line) and the modified version (solid line) when $\epsilon_{e f}^{e}=$ $\epsilon_{e f}^{o}$.

deteriorates drastically (this has been both simulated and measured, although results are not included for the sake of space saving). This phenomenon is closely related to the excitation of the slot mode in the coplanar-waveguide version of parallel-coupled line filters. Air bridges are required to suppress the undesired mode in such a case [17] because of the coplanar nature of the structure, but thanks to our double sided geometry, metal bridges are just a part of the ground-plane pattern. Fig. 4 depicts the simulated responses for the conventional and modified filters (assuming $\epsilon_{e f}^{e}=\epsilon_{e f}^{o}$ ). Important spurious band reduction is clearly observed. In Fig. 5, we compare Ensemble results for the final design (row 3 in Table II) and the measurements of the fabricated filter. Although the spurious band is not completely removed (due to imprecision in the filter dimensions associated to our fabrication process), its level is below $-25 \mathrm{~dB}$.

\section{Two-Pole Chebyshev Filter of $\Delta=20 \%$ and Ripple $0.5 \mathrm{~dB}$}

Here, as an example of the second strategy mentioned in Section I, we have designed a Chebyshev bandpass filter of parameters $f_{0}=2 \mathrm{GHz}, \Delta=20 \%$, order $N=2$, and ripple $0.5 \mathrm{~dB}$. In this case, the classical design provides the values $Z_{\text {even }}=84.86 \Omega$ and $Z_{\text {odd }}=37.54 \Omega$ for Sections 1 and 3 and $Z_{\text {even }}=70.75 \Omega$ and $Z_{\text {odd }}=39.20 \Omega$ for Section II. Dimensions for a conventional filter and modified filter implemented in the substrate of cases A and B are shown in Table III (no edge effects are accounted for). Fig. 6 shows the simulated filter responses for filters in Table III. As expected, the spurious band of the modified filter is significantly reduced and the central frequency is slightly shifted. To completely remove the spurious band, we have widen the slots only in the neighborhood of the transitions between filter sections, such as shown in Fig. 7 (dimensions of perturbed slots are noted

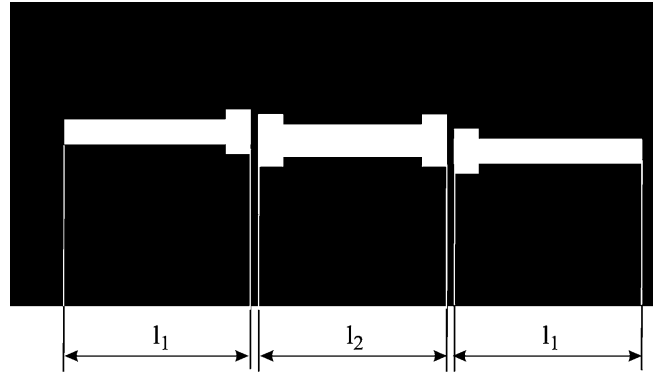

Fig. 7. Local perturbation of the slots in the ground plane to completely remove the spurious band (case filter $\mathrm{C}$ ). The sections lengths to achieve the desired central frequency are $l_{1}=14.83 \mathrm{~mm}$ and $l_{2}=14.68 \mathrm{~mm}$. The slots are $1 \mathrm{~mm}$ wider along a distance of $2.15 \mathrm{~mm}$.

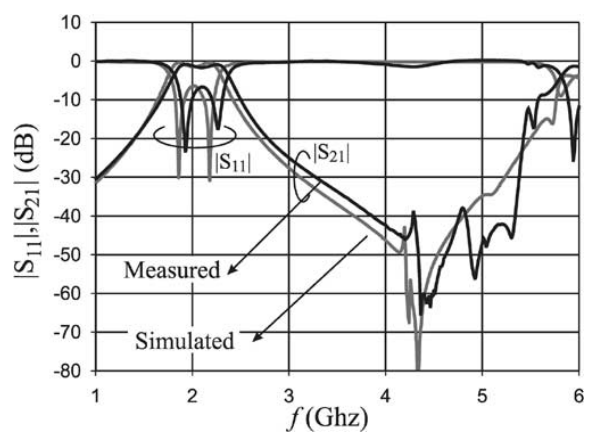

Fig. 8. Comparison between simulated and measured results of the final version of filter $\mathrm{C}$.

in the caption). Note that, except in this zone, the slot has the dimensions that make the even and odd modal phase velocities equal.

The results of the simulation with Ensemble for the final version of the filter with locally perturbed slots are compared with the measurements in Fig. 8. Once again, it can be appreciated that the spurious band practically disappears and that the agreement between experimental and simulated results can be considered acceptable. Note that local perturbation of the slot width is enough for modal electrical lengths compensation because the main cause of mismatching (different even/odd-mode phase velocities) has been already removed by proper adjustment of $s_{R}$. Local slot widening is just a fine adjustment that is not critical. Just a few full-wave simulations are enough to properly tune the dimensions of the local perturbation.

\section{CONCLUSIONS}

A simple method to remove the spurious band of conventional PCMFs has been presented. The width of a slot practiced in the ground plane along each coupled section has been adjusted to compensate for unequal modal electrical lengths. The preliminary design neglecting end effects has been based on a fast quasi-TEM approach. These effects are then accounted for by using a full-wave simulator for planar circuits and compensated either by fine tuning of the ground-plane slot widths or by local perturbation of such slots. Experimental verification of the theory is provided. A satisfactory agreement between simulated and measured responses has been found. 


\section{REFERENCES}

[1] S. B. Cohn, "Parallel-coupled transmission-line resonator filters," IRE Trans. Microwave Theory Tech., vol. MTT-6, pp. 223-231, Apr. 1958.

[2] J.-S. Hong and M. J. Lancaster, Microstrip Filters for RF/Microwave Applications. New York: Wiley, 2001.

[3] I. J. Bahl, "Capacitively compensated high performance parallel coupled microstrip filters," in IEEE MTT-S Int. Microwave Symp. Dig., 1989, pp. 679-681.

[4] S. Denis, C. Person, S. Toutain, B. Théron, and S. Vigneron, "Parallel coupled microstrip filter with phase difference compensation," Electron. Lett., vol. 31, no. 22, pp. 1927-1928, Oct. 1995.

[5] C. Person, A. Sheta, J. Coupez, and S. Toutain, "Design of high performance band pass filters by using multilayer thick film technology," in 24th Eur. Microwave Conf., vol. 1, 1994, pp. 446-471.

[6] J. T. Kuo and M. Jiang, "Suppression of spurious resonance for microstrip bandpass filters via substrate suppression," in Asia-Pacific Microwave Conf., Kyoto, Japan, 2002, pp. 497-500.

[7] M. Le Roy, A. Perennec, S. Toutain, and L. C. Calvez, "Continuously varying coupled transmission lines applied to design band-pass filter," Int. J. RF Microwave Computer-Aided Eng., vol. 12, no. 3, pp. 288-295, May 2002.

[8] J. T. Kuo and W. Hsu, "Parallel coupled microstrip filters with suppression of harmonic response," IEEE Microwave Wireless Comp. Lett., vol. 12 , pp. 383-385, Oct. 2002.

[9] C.-Y. Chan and T. Itoh, "A modified parallel-coupled filter structure that improves the upper stopband rejection and response symmetry," IEEE Trans. Microwave Theory Tech., vol. 39, pp. 310-313, Feb. 1991.

[10] S. Llorente, C. S. Segundo, A. Gutiérrez, and M. Salazar-Palma, "Filtro de líneas acopladas con ceros de transmisión en tecnología microstrip" (in Spanish), in XUII URSI Nat. Symp., Alcalá de Henares, Spain, 2002, pp. 313-314

[11] F.-R. Yang, K.-P. Ma, Y. Qian, and T. Itoh, "A uniplanar compact photonic-bandgap (UC-PBG) structure and its applications for microwave circuits," IEEE Trans. Microwave Theory Tech., vol. 47, pp. 1509-1514, Aug. 1999.

[12] T. Lopetegi, M. A. Laso, J. Hernández, M. Bacaicoa, D. Benito, M. J. Garde, M. Sorolla, and M. Guglielmi, "New microstrip wiggly-line filters with spurious passband suppression," IEEE Trans. Microwave Theory Tech., vol. 49, pp. 1593-1598, Sept. 2001.

[13] L. Zhu, H. Bu, and K. Wu, "Broadband and compact multi-pole microstrip bandpass filters using ground plane aperture technique," Proc. Inst. Elect. Eng., pt. H, vol. 149, no. 1, pp. 71-77, Feb. 2002.

[14] F. Masot, F. Medina, and M. Horno, "Theoretical and experimental study of modified coupled strip coupler," Electron. Lett., vol. 28, no. 4, pp. 347-348, Feb. 1992.

[15] J. Martel and F. Medina, "A suitable integral equation for the quasi-TEM analysis of hybrid strip/slot-like structures," IEEE Trans. Microwave Theory Tech., vol. 49, pp. 224-228, Jan. 2001.

[16] C. Nguyen, "Accurate equation for determining resonator length in halfwavelength parallel-coupled bandpass filter," Electron. Lett., vol. 29, no. 6, pp. 532-533, Mar. 1993

[17] F. Mernyei, I. Aoki, and H. Matsuura, "MMIC bandpass filter using parallel-coupled CPW lines," Electron. Lett., vol. 30, no. 22, pp. 1862-1863, Oct. 1994.

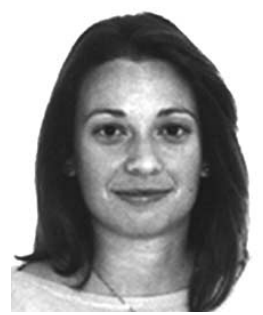

Maria del Castillo Velazquez-Ahumada was born in Lebrija, Sevilla, Spain, in 1976. She received the Licenciado degree in physics from the Universidad de Sevilla, Seville, Spain, in 2001, and is currently working toward the Ph.D. degree in electronics and electromagnetism from the Universidad de Sevilla.

Her research is focused upon printed passive microwave filters and couplers.

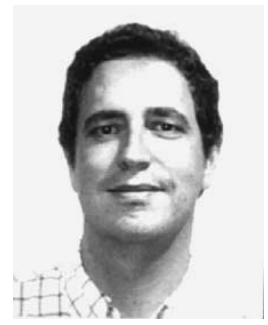

Jesús Martel was born in Seville, Spain, in 1966. $\mathrm{He}$ received the Licenciado and Doctor degrees in physics from the Universidad de Sevilla, Seville, Spain, in 1989 and 1996, respectively.

Since 1992, he has been with the Grupo de Microondas, Departamento de Física Aplicada 2, Escuela Tecnica Superior (ETS) de Arquitectura, Universidad de Sevilla, where, in 2000, he became an Associate Professor. His current research interest is focused on the numerical analysis of planar transmission lines, modeling of planar microstrip discontinuities, design of passive microwave circuits, microwave measurements, and artificial media.

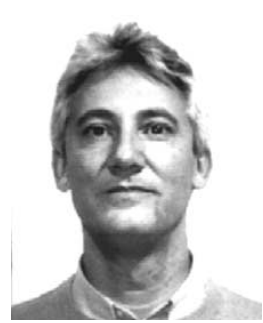

Francisco Medina (M'90-SM'01) was born in Puerto Real, Cádiz, Spain, in November 1960. He received the Licenciado and Doctor degrees from the Universidad de Sevilla, Seville, Spain, in 1983 and 1987, respectively, both in physics.

From 1986 to 1987, he spent the academic year with the Laboratoire de Microondes de l'ENSEEIHT, Toulouse, France. From 1985 to 1989, he was a Profesor Ayudante (Assistant Professor) with the Departamento de Electrónica y Electromagnetismo, Universidad de Sevilla, and since 1990, he has been a Profesor Titular (Associate Professor) of electromagnetism. He is also currently Head of the Grupo de Microondas, Universidad de Sevilla. His research interest includes analytical and numerical methods for guidance, resonant and radiating structures, passive planar circuits, and the influence on these circuits of anisotropic materials. He is reviewer of a number of international scientific and technical journals and local conferences.

Dr. Medina was a member of the Technical Program Committee (TPC) of the 23rd European Microwave Conference, Madrid, Spain, 1993, the TPC of ISRAMT, Malaga, Spain, 1999, and the TPC of the Microwaves Symposiun, Tetouan, Morocco, 2000. He is on the Editorial Board of the IEEE TRansactions on Microwave Theory and TeChNiques. He has been a reviewer for other IEEE and Institution of Electrical Engineers (IEE), U.K., publications. He was the recipient of a Ministerio de Educación y Ciencia/Ministere de la Recherche et la Technologie Scholarship. 